\title{
Circulating microRNAs are deregulated in overweight/obese children: preliminary results of the I.Family study
}

\author{
Giuseppe lacomino ${ }^{1 *}$, Paola Russo ${ }^{1}$, Ilaria Stillitano1, Fabio Lauria', Pasquale Marena', Wolfgang Ahrens ${ }^{2,3}$, \\ Pasquale De Luca ${ }^{4}$ and Alfonso Siani ${ }^{1}$
}

\begin{abstract}
Background: MicroRNAs (miRNAs) are small non-coding RNAs involved in the modulation of gene expression and in the control of numerous cell functions. Alterations of miRNA patterns frequently occur in cancer and metabolic disorders, including obesity. Recent studies showed remarkable stability of miRNAs in both plasma and serum making them suitable as potential circulating biomarkers for a variety of diseases and conditions.

The aim of this study was to assess the profile of circulating miRNAs expressed in plasma samples of overweight or obese (OW/Ob) and normal weight (NW) prepubertal children from a European cohort (www.ifamilystudy.eu). The project, aimed to assess the determinants of eating behavior in children and adolescents of eight European countries, is built on the IDEFICS cohort (www.ideficsstudy.eu), established in 2006. Among the participants of the I.Family Italian Cohort, ten OW/Ob (age $10.7 \pm 1.5$ years, BMl $31.6 \pm 4.3 \mathrm{~kg} / \mathrm{m}^{2}$ ) and ten NW (age $10.5 \pm 2.7$ years, BMl $16.4 \pm 1.7 \mathrm{~kg} / \mathrm{m}^{2}$ ) children were selected for the study. Gene arrays were employed to differentially screen the expression of 372 miRNAs in pooled plasma samples. Deregulated miRNAs $(p<0.05)$ were further validated in the individual samples using a real-time PCR (RT-qPCR) approach.

Results: Using a significance threshold of $p<0.05$ and a fold-change threshold of \pm 4.0 , we preliminarily identified in the pooled samples eight miRNAs that differed between the OW/Ob and NW groups. The validation by RT-qPCR in the individual plasma samples showed a twofold upregulation of miR-31-5p, a threefold upregulation of miR-2355-5p, and a 0.5-fold downregulation of miR-206 in OW/Ob as compared with NW. The molecular functions of these differentially expressed plasma miRNAs as well as their expected mRNA targets were predicted by bioinformatics tools.
\end{abstract}

Conclusions: This pilot study shows that three circulating miRNAs are differentially regulated in OW/Ob as compared with NW children. Although causal pathways cannot be firmly inferred by these results, that deserve confirmation in larger samples, it is conceivable that circulating miRNAs may be novel biomarkers of obesity and related metabolic disturbances.

Keywords: Circulating miRNAs, Childhood obesity, Metabolic disorders, Biomarker

\section{Background}

The obesity epidemic represents a major health challenge worldwide since it is associated with severe adverse consequences for human health [1-3]. Excess energy intake and lack of physical activity are the main factors that drive the development of obesity. Individual traits, such as the genetic profile, likewise contribute to

\footnotetext{
* Correspondence: piacomino@isa.cnr.it

'Institute of Food Sciences, CNR, Via Roma, 64, 83100 Avellino, Italy

Full list of author information is available at the end of the article
}

obesity. A quantity of studies has found a number of genes associated with obesity and obesity-related phenotypes [4-6].

Adipose tissue, the storage site of triglycerides, acts as an endocrine organ contributing to regulate energy homeostasis $[7,8]$. Anomalous fat accumulation in obesity increases the risk of severe diseases such as metabolic syndrome, type 2 diabetes, atherosclerosis, and cancer [9]. Evidence suggests that in obesity, adipose tissues stay in a state of subclinical chronic inflammation [10] 
mainly prompted by the massive recruitment of macrophages into adipose tissues [11]. Adipogenesis is tightly controlled by a mixture regulatory signals including endocellular transcription factors and circulating hormones [12]. Additional regulators of adipogenesis also include microRNAs (miRNAs) [13-17]. These small non-coding RNAs, which have a length of only 20-24 nucleotides, are involved in the modulation of gene expression and, consequently, in the control of numerous cell functions [18-20]. At present, more than 2.000 different miRNAs have been described in humans and their number is still increasing (miRBase) (http://www.mirbase.org). miRNAs are important elements of the cell epigenetic machinery that, opposing to the other known players of epigenetic regulation (DNA methylation and post-translational histone modifications), can affect gene expression by binding to the $3^{\prime}$ untranslated sequence of a target messenger RNA (mRNA) [21]. Each miRNA can target many transcripts, and transcription levels of a gene may be regulated by multiple miRNAs [21, 22]. Computational and experimental analyses indicate that endogenous miRNAs regulate the expression of up to $30 \%$ of mouse and human genes [23]. miRNAs have been shown to be involved in fundamental cellular processes including proliferation, differentiation, DNA repair, apoptosis, and metabolism [24, 25].

As a consequence, remarkable evidence indicates that dysregulation of miRNAs is causative and/or indicator of a number of disease processes including cancer [26]. Altered circulating miRNA profiles have been linked to numerous cardiometabolic diseases, including hypertension (let-7e), type 2 diabetes (miR-126), hepatic injury (miR-122), and atherosclerosis (miR-223) [14].

The presence of extracellular circulating miRNAs in blood and other biological fluids is well established $[27,28]$, but the reason for the nuclease resistance of miRNAs outside the cell remained unclear for a long period [29]. The discovery that cell-free miRNAs are detectable in serum and plasma and that their expression varies as a result of disease offers great potential for the discovery of novel health/disease biomarkers and candidate therapeutic targets [30].

Various miRNAs have been recognized to play a role in adipogenesis [31]. Preliminary evidence showed that some circulating miRNAs are associated with obesity and related metabolic disturbances in adults as well as in children (see $[16,32]$ for review).

The primary aim of our study was to identify circulating miRNAs potentially associated to early obesity via an integrated study comprising miRNA signatures and bioinformatic analyses in children participating to the I.Family survey. Here, we present the results of a pilot study on a relatively small sample of children belonging to the Italian cohort of the I.Family survey.

\section{Results and discussion}

\section{Distinct miRNA expression in plasma of OW/Ob and NW} children

The present study was aimed to identify new insights into the pathogenesis of childhood obesity according to the experimental scheme reported in Fig. 1. The main characteristics of the participants are described in Table 1. As time saving and efficiency strategy, samples from ten overweight or obese $(\mathrm{OW} / \mathrm{Ob})$ and ten normal weight $(\mathrm{NW})$ were analyzed as pools in triplicate by $\mathrm{PCR}$ arrays.

The geometric mean $\mathrm{Ct}$ value of multiple selected housekeeping genes was 26.13 for NW and 26.38 for OW/Ob. Reverse transcription control average $C_{t}$ (RTC) was 20.68 for NW and 20.75 for OW/Ob. Absent calls were $1.82 \%$ for NW and $2.34 \%$ for OW/Ob.

Using a significance threshold of $p<0.05$ and a foldchange threshold of \pm 4.0 , we identified in the pooled samples eight out of 372 total screened miRNAs that differed between the $\mathrm{OW} / \mathrm{Ob}$ and NW groups (Table 2). A scatter plot (Fig. 2) shows the relative expression levels of miRNAs in OW/Ob vs. NW pooled plasma samples.

The eight miRNAs found to be differentially expressed in the pooled analysis were subsequently validated in real-time PCR (RT-qPCR) as distinct assays performed in triplicate in the 20 individual plasma samples. PCR reference gene included the endogenous spike-in C.elmiR-39-3p. As reported in Table 3, the differential expression of three miRNAs was statistically confirmed ( $p$ value $<0.05$ ): a twofold upregulation of miR-31-5p, a threefold upregulation of miR-2355-5p, and a 0.5-fold downregulation of miR-206 were observed in OW/Ob as compared with NW.

\section{miRNAs target prediction, KEGG pathway analysis}

The specialized algorithm miRPath v2.0 [33] was used to understand the molecular functions of deregulated miRNAs and to predict their putative mRNA targets. To recognize biologically relevant molecular networks and pathways, we further explored miRNA targets by analyzing molecular interactions on the comprehensive knowledge base including the Kyoto Encyclopedia of Genes and Genomes (KEGG) [34] and Ingenuity Pathways Analysis. Both IPA and KEGG technologies provided hints for the functions of miRNAs.

Top pathways predicted to be actively regulated by miRNAs were classified according to the KEGG functional annotation (Table 4).

Results of mir-31-5p MiRPath analysis on experimentally validated miRNA interactions (derived from DIANA-TarBase v6.0) or predicted targets (provided by the DIANA-microT-CDS algorithm) are reported in Table 5. 


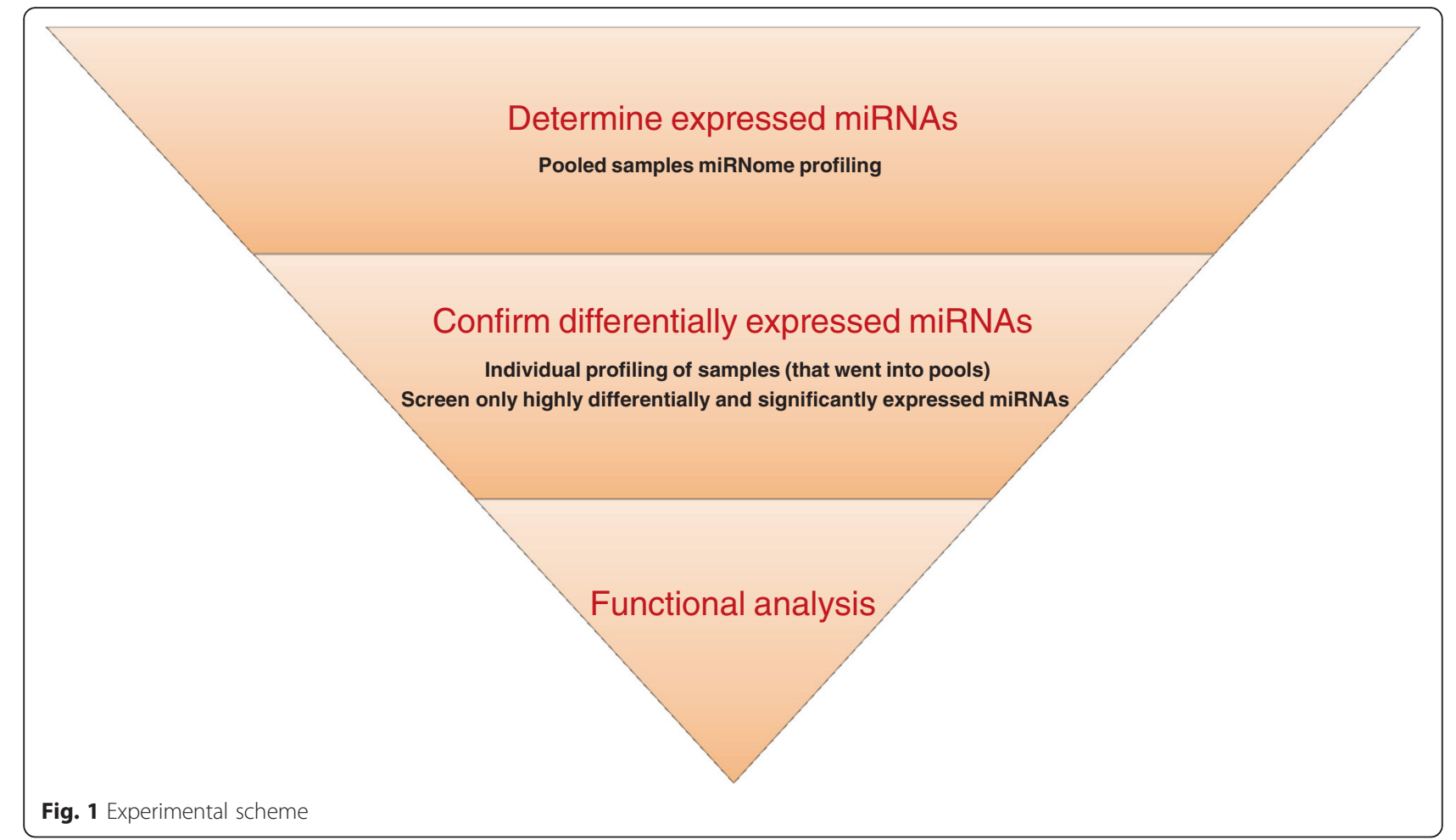

Computational prediction of miR-31 identified the following targets in the "fatty acid degradation" pathway: (a) the enoyl-CoA hydratase, a key enzyme in the breakdown of fatty acids essential to metabolizing fatty acids to produce both acetyl CoA and energy, and (b) the alcohol dehydrogenase 1A (Additional file 1: Figure S1).

Among the top predicted targets of miR-31-5p, we also identify the CCAAT-enhancer-binding protein- $\alpha$ $(\mathrm{C} / \mathrm{EBP} \alpha)$, a main modulator of the expression of genes involved in cell cycle regulation as well as adipocyte functions during adipogenesis (Fig. 2).

As relevant miR-206 targets, we recognized the "insulin signaling," the "pentose phosphate" pathways, and the

Table 1 Anthropometric and metabolic variables of the individuals included in this study

\begin{tabular}{llll}
\hline & Normal weight & Overweight/Obese & $p$ \\
\hline Sex (M/F) & $5 / 5$ & $6 / 4$ & \\
Age (years) & $10.50 \pm 2.76$ & $10.70 \pm 1.57$ & n.s \\
BMl (kg/cm²) & $16.45 \pm 1.71$ & $31.68 \pm 4.32$ & $<0.001$ \\
Trg (mg/dL) & $53.80 \pm 22.08$ & $84.20 \pm 30.01$ & 0.02 \\
TC (mg/dL) & $146.80 \pm 24.10$ & $163.40 \pm 33.87$ & n.s \\
HDL (mg/dL) & $62.30 \pm 12.33$ & $52.10 \pm 12.37$ & n.s \\
LDL (mg/dL) & $77.60 \pm 22.51$ & $100.70 \pm 30.57$ & n.s \\
Glu (mg/dL) & $91 \pm 6.16$ & $94.6 \pm 3.53$ & n.s \\
\hline
\end{tabular}

Value are mean \pm standard deviation n.s $=$ not significant liver X receptor- $\alpha(\mathrm{LXR} \alpha)$, a ligand-dependent transcription factor playing a relevant role in the metabolism and homeostasis of lipids, cholesterol, bile acids, and steroid hormones with a preeminent expression in the liver.

Further, we identified the oleoyl-ACP hydrolase, a medium-chain acyl-[acyl-carrier-protein] hydrolase in the "fatty acid biosynthesis" pathway as a target of miR$2355(p=0.005)$.

Finally, the target prediction of concurrently deregulated miRNAs gave evidence for the "Notch" signaling $(p=0.0008)$ (Additional file 2: Figure S2) and the

Table 2 Differential miRNA expression profile determined by gene-arrays screening

\begin{tabular}{lcr}
\hline miRNA & Fold regulation & \\
\hline miR-26b-5p & 25.3723 & $\uparrow$ \\
miR-31-5p & 4.9499 & $\uparrow$ \\
miR-2355-5p & 6.5216 & $\uparrow$ \\
miR-1231 & -8.7217 & $\downarrow$ \\
miR-361-3p & -4.8918 & $\downarrow$ \\
miR-136-5p & -4.8356 & $\downarrow$ \\
miR-320a & -9.9692 & $\downarrow$ \\
miR-206 & -6.0515 & $\downarrow$
\end{tabular}

miRNAs showing significant differences in expression levels between the compared groups are reported. Analyses were generated setting the threshold at \pm 4 and $p$ value $<0.05$

up arrow $=$ upregulated; down arrow $=$ downregulated 


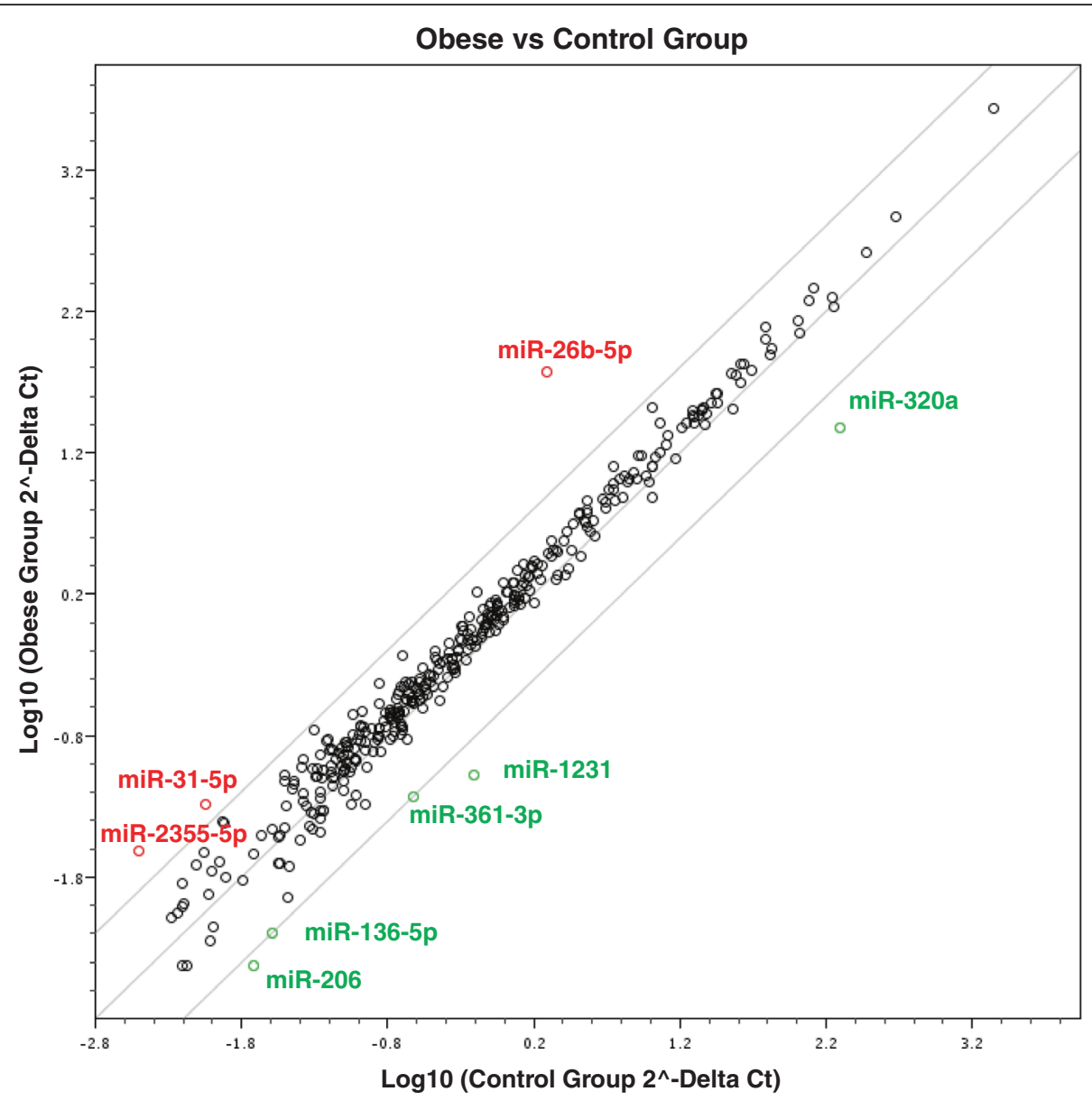

Fig. 2 Differential expression of circulating miRNAs in plasma. Comparisons of OW/Ob vs. NW circulating miRNA profiles. The expression levels were queried by qPCR arrays. Means of each data point are presented as a scatter plot ( $n=3$ in each group). Selection threshold is \pm 4.0 . Upregulated miRNAs are marked red whereas downregulated miRNAs are marked green

"Arrhythmogenic right ventricular cardiomyopathy" pathways $\left(p=2.905898 \times 10^{-05}\right)$ (Additional file 3: Figure S3).

\section{Discussion}

We identified three circulating miRNAs differentially expressed in plasma samples of OW/Ob children. Bioinformatic exploratory analysis predicted their involvement in critical pathways including lipid metabolism and adipocyte differentiation.

Table 3 Differential miRNA profile confirmed by RT-qPCR

\begin{tabular}{llllll}
\hline miRNA & OW/Ob & NW & $\begin{array}{l}\text { Fold } \\
\text { regulation }\end{array}$ & $p$ & \\
\hline miR-31-5p & $3.60(3.42-3.97)$ & $1.86(0.47-2.40)$ & 1.92 & 0.0003 & $\uparrow$ \\
miR-2355-5p & $3.65(2.52-5.35)$ & $1.23(0.21-1.78)$ & 2.93 & 0.0166 & $\uparrow$ \\
miR-206 & $1.28(0.63-1.83)$ & $2.45(1.29-4.92)$ & 0.52 & 0.0461 & $\downarrow$ \\
\hline
\end{tabular}

Differential miRNAs were confirmed by RT-qPCR. Reported values are mean (minimum-maximum). Fold regulation was expressed as fold change with respect to non-obese controls. Reference included the endogenous spike-in C.el-miR-39-3p

up arrow $=$ upregulated; down arrow $=$ downregulated
Table 4 MiRPath analysis

\begin{tabular}{ll}
\hline KEGG pathway & $p$ value \\
\hline Gap junction & $1.13 \mathrm{E}-13$ \\
Arrhythmogenic right ventricular cardiomyopathy (ARVC) & $2.90 \mathrm{E}-05$ \\
Notch signaling pathway & 0.0003 \\
Regulation of actin cytoskeleton & 0.0005 \\
Dorso-ventral axis formation & 0.0008 \\
Bacterial invasion of epithelial cells & 0.0009 \\
Axon guidance & 0.0026 \\
Shigellosis & 0.0200 \\
Endocrine and other factor-regulated calcium reabsorption & 0.0397 \\
Glycosaminoglycan biosynthesis-heparan sulfate/heparin & 0.0483 \\
Pathways in cancer & 0.0490 \\
Thyroid cancer & 0.0500
\end{tabular}

Target genes were classified according to KEGG functional annotations to identify top pathways that were actively regulated by miRNAs. Merged $p$ value is extracted by combining calculated significance levels using Fisher's meta-analysis method 
Table 5 miRPath analysis of Hsa-mir-31-5p interactions

\begin{tabular}{|c|c|c|}
\hline $\begin{array}{l}\text { (A) KEGG-predicted pathway derived from } \\
\text { DIANA-microT-CDS database }\end{array}$ & $p$ value & genes \\
\hline Metabolism of xenobiotics by cytochrome P450 & $1.46 \mathrm{E}-07$ & 3 \\
\hline Inositol phosphate metabolism & 0.00442 & 4 \\
\hline Fatty acid metabolism & 0.02773 & 2 \\
\hline $\begin{array}{l}\text { (B) KEGG-predicted targets provided by the } \\
\text { DIANA-TarBase v6.0 database }\end{array}$ & $p$ value & genes \\
\hline Regulation of actin cytoskeleton & $6.24 \mathrm{E}-06$ & 5 \\
\hline Bacterial invasion of epithelial cells & $1.79 \mathrm{E}-05$ & 3 \\
\hline Axon guidance & 8.85E-05 & 3 \\
\hline Shigellosis & 0.00062 & 2 \\
\hline Pathogenic Escherichia coli infection & 0.00276 & 2 \\
\hline Chemokine signaling pathway & 0.00653 & 3 \\
\hline Pertussis & 0.00746 & 2 \\
\hline Hypertrophic cardiomyopathy (HCM) & 0.00979 & 2 \\
\hline Dilated cardiomyopathy & 0.01157 & 2 \\
\hline T cell receptor signaling pathway & 0.01604 & 2 \\
\hline Leukocyte transendothelial migration & 0.01604 & 2 \\
\hline $\begin{array}{l}\text { Arrhythmogenic right ventricular cardiomyopathy } \\
\text { (ARVC) }\end{array}$ & 0.01604 & 2 \\
\hline Dorso-ventral axis formation & 0.03214 & 1 \\
\hline
\end{tabular}

miRPath analysis of miR-31-5p (A) of predicted targets provided by the DIANAmicroT-CDS algorithm or (B) of experimentally validated miRNA interactions derived from the DIANA-TarBase v6.0. Target pathways were classified according to KEGG functional annotations

Adipose tissue is involved in pathophysiological processes because it influences metabolism. In adipogenesis, pluripotent adipose-derived mesenchymal stem cells differentiate into adipocytes. This process is finely regulated by both hormones and transcription factors in a complex signaling network. A number of miRNAs and their targets were recently identified to play regulatory roles in adipocyte differentiation [35-37]. To date, up to 221 miRNAs have been found to be expressed or dysregulated in adipose tissue or adipocytes in mammals [16].

Differentiation from pre-adipocytes into mature adipocytes is orchestrated by several transcription factors such as peroxisome proliferator-activated receptor- $\gamma$ (PPAR $\gamma$ ) and $\mathrm{C} / \mathrm{EBPs}(\mathrm{C} / \mathrm{EBPs}) . \mathrm{C} / \mathrm{EBP} \beta$ and $\mathrm{C} / \mathrm{EBP} \delta$ are induced by adipogenic stimuli and represent primary regulators of adipogenesis. Targets of $C / E B P \beta$ and $C / E B P \delta$ are the promoters of the genes encoding crucial adipogenic factors as C/EBP $\alpha$ and PPAR $\gamma$ as well as the sterol regulatory element-binding protein (SREBP1), the key regulator of lipogenic genes. The protein encoded by the $\mathrm{C} / \mathrm{EBP} \alpha$ intronless gene is a leucine zipper transcription factor which can bind to specific promoters and enhancers as a homodimer or it can form heterodimers with the related proteins $\mathrm{C} / \mathrm{EBP} \beta$ and $\mathrm{C} / \mathrm{EBP} \gamma$ (Fig. 3).
The C/EBP $\alpha$ has been shown to bind to the promoter and to also modify the expression of the leptin gene that plays a central role in body weight homeostasis. C/EBP $\alpha$ is sufficient to trigger differentiation of pre-adipocytes into mature adipocytes [38]. Peculiarly, PPAR $\gamma$ directly triggers endogenous $\mathrm{C} / \mathrm{EBP} \alpha$ transcription. In turn, $\mathrm{C} /$ EBP $\alpha$ activates the PPAR $\gamma$ gene through a positive feedback loop and thereby promotes adipogenesis [12]. Concurrently, PPAR $\gamma$ and $\mathrm{C} / \mathrm{EBP} \alpha$ induce the expression of genes that are involved in insulin sensitivity, lipogenesis, and lipolysis and, ultimately, in terminal differentiation and mature functions of adipocytes.

miR-31 directly targets C/EBP $\alpha$ transcript whose levels are in vivo downregulated by miR-31 [37]. Notably, miR-31 expression is inversely correlated with adipogenic differentiation status [39], and consequently, it has a decisive anti-adipogenic function.

We found that miR-31 was significantly upregulated in plasma of $\mathrm{OW} / \mathrm{Ob}$ children identifying $\mathrm{C} / \mathrm{EBP} \alpha$ as a privileged target of miR-31. miR-31 upregulation may be considered as an apparent contradiction, but it probably reflects the well-established role of circulating miRNAs as "endocrine signals." The concept that miRNAs may be transferred from donor to recipient cells is now well established with the relevant implication that miRNAs could affect gene expression distally, so acting as paracrine or endocrine signaling molecules. Accordingly, a panel of "endocrine miRNAs" released from fat cells that may be used as a marker of disturbed adipose tissue function has been characterized [16]. The idea that miR-31 acts as an obesity controlling signal seems to be supported by the literature. Actually, in analyzing the expression of miRNAs in the livers of leptindeficient obese (ob/ob) mice compared to normal C57BL/6 mice, Li et al. found that ob/ob mice exhibited an upregulation of miR-31 [40], despite its wellestablished anti-adipogenic role.

Computational prediction of miR-31 recognized several targets in the "fatty acid degradation" pathway $(p=0.027)$ (Additional file 1: Figure S1). Whether miR-31 deregulation plays a causative role or is merely an epiphenomenon in the etiology of obesity still remains to be determined.

In this study, we also observed that miR-206 was significantly downregulated in plasma of $\mathrm{OW} / \mathrm{Ob}$ children. miR-206 belongs to the cluster of the so-called myomiRs and represents one of the most studied tissue-specific miRNAs involved in skeletal muscle differentiation. Several studies indicate that miR-206 plays a key role in the growth and development of skeletal muscle, promoting myogenic differentiation [41]. Moreover, it has been well established that it is related to the pathogenesis of numerous diseases including heart failure, chronic obstructive pulmonary disease, and Alzheimer's disease 


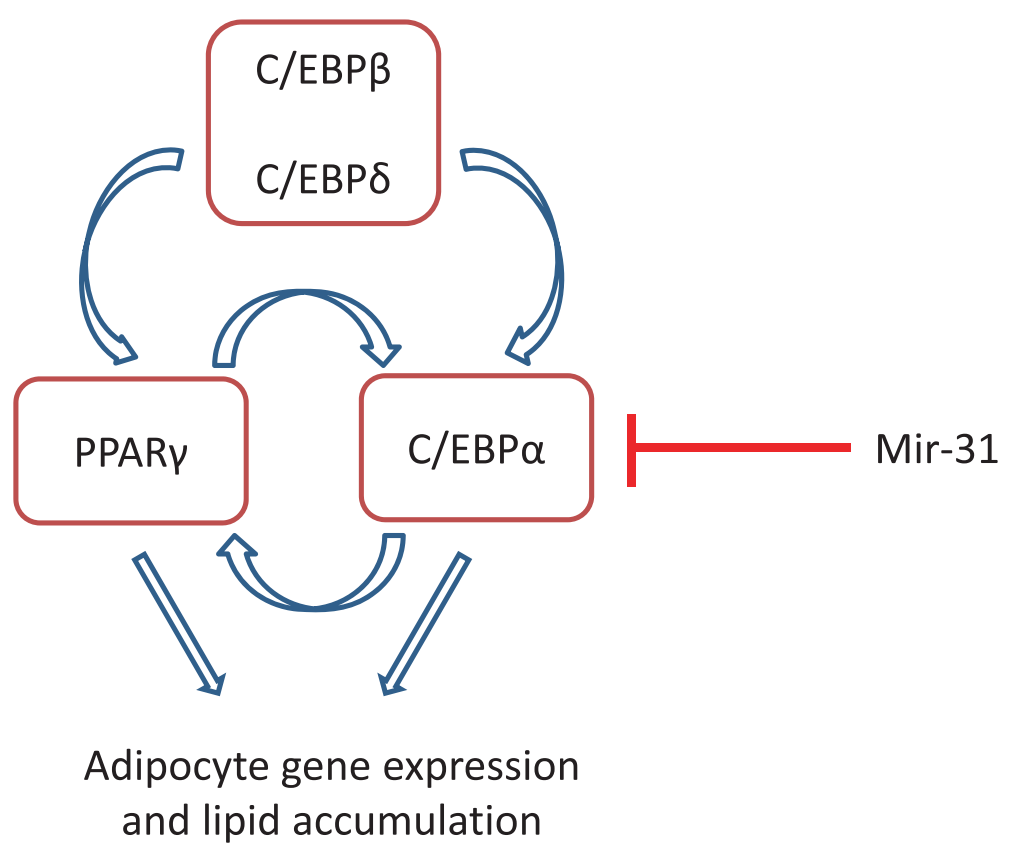

Fig. $3 \mathrm{C} / \mathrm{EBPa}$, a key target. The C/EBPa intronless gene encodes a transcription factor containing a basic leucine zipper domain. The protein acts in homodimers or heterodimers that recognize the CCAAT motifs in the promoters of target genes. Complexes modulate the expression of genes involved in cell cycle regulation as well as adipocyte functions: during adipogenesis highly induced genes are synergistically controlled by both PPARY and C/EBPa

and in numerous kinds of cancers. Interestingly, in most of these diseases, miR-206 levels are downregulated, which leads to the recognition of miRNA as a "diseaseavoiding" molecule. Moreover, miR-206 expression is abundant in brown adipocytes but missing in white adipocytes [42]. Remarkably, it has been shown that miR206 suppresses liver LXR $\alpha$, a gene target of PPAR, consequently inhibiting lipogenesis and controlling lipid metabolism [43]. The insulin signaling pathway $(p=0.01,11$ target genes) and the pentose phosphate pathway ( $p=0.01,4$ target genes) were also identified as candidate targets of miR-206. Therefore, the reduced level of circulating miR-206 in OW/Ob children may be either representative of a functional decreased synthesis [42] or an epiphenomenon reflecting the reduced relative skeletal muscle mass in the $\mathrm{OW} / \mathrm{Ob}$ group.

Among the top pathways that were predicted to be targeted by the three differentially expressed miRNAs, "Notch" signaling appears relevant $(p=0.0008,5$ target genes, Additional file 2: Figure S2). Notch signaling represents a basic biological pathway primarily related to cell communication and development with extensive integration and crosstalk with other signaling pathways. Perturbations in this pathway have been linked to many genetic disorders and cancers. Accordingly, the direct involvement of Notch signaling has recently been demonstrated in obesity and type 2 diabetes [44]: through transcriptional binding partners FoxO1 and Rbpj, Notch activation enhances pathologic glucose output and fatty liver in obese, in combination with a reduced Akt activation by insulin. Activation of the Notch pathway in obesity results in heightened diabetes and fatty liver production, as a consequence of an augmented lipogenesis that is not blocked despite the reduced insulin signaling. Notably, blocking Notch signaling in mice caused white fat cells to transform into beige fat consequently reducing the risk of obesity and related health problems [44].

While we recognize the limitations and the relative reliability of our target prediction analyses, this hypothesis-generating exercise suggested the involvement of the detected miRNAs in metabolic relevant pathways, to be obviously confirmed by larger and ad hoc designed studies.

Our results confirm those of recent papers indicating that circulating miRNAs are deregulated in obese children [45] and are associated with obesity and lipid abnormalities in adolescents [46]. However, differentially detected miRNA signals in refs. 46 and 47 are not consistent with those identified in our study. Of note, all these miRNAs are present in the 372 miRNA arrays we used in our study but did not pass the threshold we set to consider them as differentially expressed. A solid explanation of these discrepancies is not at hand. Considering the rapidly changing scenario in this field, the 
interpretation and discussion of the inconsistent findings regarding the association of circulating miRNAs with obesity in children $[45,46]$ is far beyond the objective of this study. However, recent reviews extensively summarized potentiality, but also uncertainties, of the role of extracellular miRNAs as potential regulators and/or biomarkers of obesity and metabolic diseases $[16,32]$. In particular, the inconsistencies of the findings may be ascribed to inter-population differences, but even, and perhaps most likely, to methodological differences in sampling, RNA isolation, detection, and normalization [32]. The need for consistent analysis strategies and quality control has been recently discussed [47].

\section{Conclusions}

This pilot study showed that three circulating miRNAs are differentially regulated in $\mathrm{OW} / \mathrm{Ob}$ as compared with NW children. Although causal pathways cannot be firmly inferred-but only suggested-by these preliminary results, that deserve confirmation in other populations, it is conceivable that circulating miRNAs may be novel biomarkers of obesity and related metabolic disturbances.

The planned completion of the miRNA studies in the much larger sample of children from the eight European countries participating to the I.Family project will certainly contribute to the ongoing debate whether circulating miRNAs play a role in disease pathogenesis, are (early) indicators of a metabolic dysfunction, or both.

\section{Methods}

\section{Study cohort}

The I.Family project aimed to assess the determinants of eating behavior in children and adolescents of eight European countries and related health outcomes was built on the IDEFICS cohort (http://www.ideficsstudy.eu), established in 2006. We selected our sample among OW/ $\mathrm{Ob}$ and NW participants of the Italian cohort of the project. The weight status of children was defined according to age- and sex-specific BMI categories [48]. Ten OW/Ob (four females and six males, age $10.7 \pm 1.5$ years, BMI $31.7 \pm 4.3 \mathrm{~kg} / \mathrm{m}^{2}$ ) and ten NW (five females and five males, age $10.5 \pm 2.7$ years, BMI $16.4 \pm 1.7 \mathrm{~kg} / \mathrm{m}^{2}$ ) children were included in the study.

The study protocol was approved by the local Ethics Committee of the local Health Authority (ASL Avellino) and informed written parental consent was obtained for each participant.

\section{Sample processing}

Whole blood was collected according to the standard operating procedures in BD Vacutainer ${ }^{\ominus}$ blood collection tubes. The optimal approach for miRNA purification from whole blood necessitates collecting fresh blood and processes the sample as quickly as possible. Accordingly, plasma was isolated by centrifugation at $1900 \times g$ for $10 \mathrm{~min}$ at $4{ }^{\circ} \mathrm{C}$ in an Eppendorf benchtop centrifuge (Eppendorf, Germany), aliquoted into $1.5 \mathrm{~mL}$ Eppendorf tubes, and promptly stored at $-80{ }^{\circ} \mathrm{C}$, in the absence of freeze-thaw cycles, until processing.

\section{miRNA extraction, reverse transcription, and pre-screening}

Prior to miRNA extraction, spectrophotometry was carried out on plasma samples to test for hemolysis by measuring the absorbance of free hemoglobin at $414 \mathrm{~nm}$; samples with $\mathrm{OD}_{414}$ greater than 0.2 were excluded from the study. RNA was extracted from both plasma pooled samples and from individual plasma samples using the commercial column-based system miRNeasy serum/plasma Kit (Qiagen, Germany) according to the manufacturer's instructions with minor modifications. Plasma was thawed on ice and spun for $10 \mathrm{~min}$ at $13,000 \times g$ at $4{ }^{\circ} \mathrm{C}$ in an Eppendorf benchtop centrifuge (Eppendorf, Germany) to pellet any debris. An aliquot of $200 \mu \mathrm{L}$ of plasma was transferred to a new microcentrifuge tube and $800 \mu \mathrm{L}$ of a Qiazol (Qiagen) mixture, containing $1.25 \mu \mathrm{g} / \mathrm{mL}$ of MS2 bacteriophage RNA (Roche Applied Science), was added to the sample. Caenorhabditis elegans miR-39 (C.el-miR-39) was spiked into the sample $\left(5.6 \times 10^{8}\right.$ molecules $)$ before the extraction process to assess RNA recovery. A rinse step $(500 \mu \mathrm{L}$ Qiagen RPE buffer) was repeated two times. Total RNA was eluted by adding $14 \mu \mathrm{L}$ of RNase-free water to the membrane of the column and incubating for $1 \mathrm{~min}$ before centrifugation at $13,000 \times g$ for $1 \mathrm{~min}$ at room temperature. The obtained RNA was stored at $-80{ }^{\circ} \mathrm{C}$.

Complementary DNA (cDNA) was generated by means of the miScript RTII kit (Qiagen), in HiSpec Buffer with miRNA-specific stem-looped RT primers. Nine microliters of isolated RNA were reverse transcribed in $20-\mu \mathrm{L}$ reactions according to the manufacturer's recommendations. The obtained cDNA was diluted 1:5.5 in RNase-free water. This procedure was repeated three times. To identify hemolyzed samples, the levels of miR-451, highly abundant in RBCs, were preliminarily assessed in cDNA samples by qPCR. Hemolyzed samples were excluded from the analysis.

\section{Circulating miRNA profiling}

Selected samples from ten $\mathrm{OW} / \mathrm{Ob}$ and ten NW children (Table 1) were pooled, and miRNAs were extracted as described above. miRNA profiling was performed by using the Serum and Plasma 384HC miScript miRNA PCR Arrays (SABiosciences, Qiagen) to assess the expression of 372 miRNAs typically detectable in serum and plasma. The SYBR green-based qPCR assay was performed with cDNA (dil. 1:225) in 10-uL reaction volume 
using a 7900HT fast Real-Time PCR System instrument (Applied Biosystems). Reaction conditions were as follows: 15 min at $95{ }^{\circ} \mathrm{C}$ and 40 cycles of $15 \mathrm{~s}$ at $94{ }^{\circ} \mathrm{C}, 30 \mathrm{~s}$ at $60{ }^{\circ} \mathrm{C}$, and $30 \mathrm{~s}$ at $72{ }^{\circ} \mathrm{C}$ with a set of technical controls on each plate which included the spike-in control and small non-coding RNAs (SNORDs) as reference genes. References for data normalization included the spike-in C.el-miR-39-3p, SNORD61, SNORD68, SNORD72, SNORD95, and SNORD96A, which have been verified to hold relatively stable expression levels across tissues and cell types. Normalization of expression was done using the geometric mean of the endogenous controls. All assays were inspected for distinct melting curves, and the Tm was checked. The amplification efficiency was also calculated. Ct values $>35$ were considered as negative amplification. Data that did not pass these criteria were omitted from further analysis. The efficiency of reverse transcription was assessed with miRTC.

Statistically significant deregulated miRNAs with a fold-change $\geq 4.0$ were selected and further validated by individual microRNA assays (SABiosciences, Qiagen) performed on the individual plasma samples of ten OW/ $\mathrm{Ob}$ and ten NW children, using the miScript SYBR green PCR kit (Qiagen) according to the manufacturer's instructions. RT-qPCR was performed in triplicates using the following conditions: $95{ }^{\circ} \mathrm{C}$ for $15 \mathrm{~min}$, followed by 40 cycles of $94{ }^{\circ} \mathrm{C}$ for $15 \mathrm{~s}, 55{ }^{\circ} \mathrm{C}$ for $30 \mathrm{~s}$, and $70{ }^{\circ} \mathrm{C}$ for $30 \mathrm{~s}$. Relative miRNA levels were determined by the ${ }^{\Delta \Delta} \mathrm{Ct}$ method using the C.el-miR-39 as endogenous normalizer.

\section{Data analysis}

On pooled samples, RT-qPCR quantifications were performed by using SDS 2.3 (Life Technologies, USA) to generate $\mathrm{Ct}$ values.

Gene Arrays data were organized in Excel (Microsoft), and the integrated web-based software package for miScript Arrays analysis was exploited. This software performs quantification using the ${ }^{\Delta \Delta} \mathrm{Ct}$ method and interpretation of the control assays. Tools are available at the SABiosciences data analysis web portal.

Data on the individual samples are presented as the average of at least three independent experiments \pm s.e.. Data Assist 3.1 software packages (Life Technologies, USA) were used to generate relative "fold-change" values. Student's $t$ test, performed with the GraphPad Prism 6 software, was used to determine the significance of any difference in the levels of miRNA expression between NW and OW/Ob subjects with alpha $<0.05$.

Obesity-associated miRNAs were tested using the well-established target prediction tool miRPath v2.0 [33]. miRPath achieves advanced analysis pipelines, such as hierarchical clustering of miRNAs and pathways based on the levels of their interactions. miRNA targets (in CDS or 3'-UTR regions) were predicted by the DIANAmicroT-CDS algorithm or even experimentally validated miRNA interactions derived from DIANA-TarBase v6.0. Predicted and/or validated interactions were subsequently combined with merging and meta-analysis algorithms.

All the predicted targets were further analyzed through the use of Kyoto Encyclopedia of Genes and Genomes (KEGG) [34] and QIAGEN's Ingenuity ${ }^{\circ}$ Pathway Analysis (IPA ${ }^{\circ}$, QIAGEN Redwood City, www.qiagen.com/ingenuity). IPA identified lists of genes that satisfy specific biological criteria scoring these genes towards pathways in the Ingenuity Knowledge Base which embraces a large database of biological and chemical relationships extracted from scientific literature.

\section{Additional files}

Additional file 1: Figure S1. In the highly predicted pathway 'Fatty acid metabolism' [hsa00071] targets of miR-31-5p are highlighted. Relevant pathways predicted to be targeted by the differentially expressed miRNAs. (PPTX $64 \mathrm{~kb}$ )

Additional file 2: Figure S2. miRNA targets are highlighted in the NOTCH signaling pathway. (PPTX $42 \mathrm{~kb}$ )

Additional file 3: Figure S3. Targets actively regulated by miRNAs in "Arrhythmogenic right ventricular cardiomyopathy" pathway. (PPTX 301 kb)

\section{Competing interests}

The authors declare that they have no competing interests.

\section{Authors' contributions}

$\mathrm{Gl}$ participated in the design of the study, performed the molecular and bioinformatic studies, executed the statistical analyses, and drafted the manuscript; PR participated in the design of the study and helped to draft the manuscript; IS performed the miRNA isolation and the molecular studies; FL helped to perform the statistical analysis and to draft the manuscript; PM carried out the sample collection and helped with the miRNA isolation; WA helped to draft the manuscript with critical revision; PDL helped to perform the molecular analyses and to draft the manuscript; AS conceived the study, participated in its design and coordination, and helped to draft the manuscript. All authors read and approved the final manuscript.

\section{Acknowledgements}

The work was done as part of the IDEFICS study (http://www.idefics.eu) and the I.Family Study (http://www.ifamilystudy.eu/). We gratefully acknowledge the financial support of the European Community within the Sixth RTD Framework Programme Contract No. 016181 (FOOD) for the IDEFICS study and within the Seventh RTD Framework Programme Contract No. 266044 for the I.Family study.

\section{Author details}

${ }^{1}$ Institute of Food Sciences, CNR, Via Roma, 64, 83100 Avellino, Italy. ${ }^{2}$ Leibniz Institute for Prevention Research and Epidemiology - BIPS, Bremen, Germany. ${ }^{3}$ Institute of Statistics, Faculty of Mathematics and Computer Science, University Bremen, Bremen, Germany. ${ }^{4}$ BioGeM, Ariano Irpino, Italy.

Received: 1 December 2015 Accepted: 8 March 2016

Published online: 21 March 2016

\section{References}

1. Zimmet P, Alberti KG, Shaw J. Global and societal implications of the diabetes epidemic. Nature. 2001;414:782-7. 
2. Kahn SE, Hull RL, Utzschneider KM. Mechanisms linking obesity to insulin resistance and type 2 diabetes. Nature. 2006;444:840-6.

3. Despres J-P, Lemieux I. Abdominal obesity and metabolic syndrome. Nature. 2006:444:881-7.

4. Berndt SI, Gustafsson S, Mägi R, Ganna A, Wheeler E, Feitosa MF, et al. Genome-wide meta-analysis identifies 11 new loci for anthropometric traits and provides insights into genetic architecture. Nat Genet. 2013:45:501-12.

5. Kunej T, Jevsinek Skok D, Zorc M, Ogrinc A, Michal JJ, Kovac M, et al. Obesity gene atlas in mammals. J Genomics. 2013;1:45-55.

6. Loos RJF, Yeo GSH. The bigger picture of FTO: the first GWAS-identified obesity gene. Nat Rev Endocrinol. 2014;10:51-61.

7. Rosen ED, Spiegelman BM. Adipocytes as regulators of energy balance and glucose homeostasis. Nature. 2006;444:847-53.

8. Schaffler A, Muller-Ladner U, Scholmerich J, Buchler C. Role of adipose tissue as an inflammatory organ in human diseases. Endocr Rev. 2006;27:449-67.

9. Kopelman PG. Obesity as a medical problem. Nature. 2000;404:635-43.

10. Kammoun HL, Kraakman MJ, Febbraio MA. Adipose tissue inflammation in glucose metabolism. Rev Endocr Metab Disord. 2014;15:31-44.

11. Kraakman MJ, Murphy AJ, Jandeleit-Dahm K, Kammoun HL. Macrophage polarization in obesity and type 2 diabetes: weighing down our understanding of macrophage function? Front Immunol. 2014;5:470.

12. Tang QQ, Lane MD. Adipogenesis: from stem cell to adipocyte. Annu Rev Biochem. 2012;81:715-36.

13. Xie H, Lim B, Lodish HF. MicroRNAs induced during adipogenesis that accelerate fat cell development are downregulated in obesity. Diabetes. 2009;58:1050-7.

14. Rottiers $\mathrm{V}$, Naar AM. MicroRNAs in metabolism and metabolic disorders. Nat Rev Mol Cell Biol. 2012;13:239-50.

15. Kim HJ, Cho H, Alexander R, Patterson HC, Gu M, Lo KA, et al. MicroRNAs are required for the feature maintenance and differentiation of brown adipocytes. Diabetes. 2014;63:4045-56.

16. Arner $P$, Kulyte A. MicroRNA regulatory networks in human adipose tissue and obesity. Nat Rev Endocrinol. 2015;11:276-88

17. Li J, Zhou C, Li J, Su Z, Sang H, Jia E, et al. Global correlation analysis for microRNA and gene expression profiles in human obesity. Pathol Res Pract. 2015;211:361-8.

18. Bartel DP. MicroRNAs: target recognition and regulatory functions. Cell. 2009;136:215-33.

19. Ghildiyal M, Zamore PD. Small silencing RNAs: an expanding universe. Nat Rev Genet. 2009;10:94-108.

20. Ha M, Kim VN. Regulation of microRNA biogenesis. Nat Rev Mol Cell Biol. 2014;15:509-24.

21. Ebert MS, Sharp PA. Roles for microRNAs in conferring robustness to biological processes. Cell. 2012;149:515-24.

22. Krek A, Grün D, Poy MN, Wolf R, Rosenberg L, Epstein EJ, et al. Combinatorial microRNA target predictions. Nat Genet. 2005;37:495-500.

23. Lewis BP, Burge CB, Bartel DP. Conserved seed pairing, often flanked by adenosines, indicates that thousands of human genes are microRNA targets. Cell. 2005;120:15-20.

24. Bartel DP. MicroRNAs: genomics, biogenesis, mechanism, and function. Cell. 2004;116:281-97

25. van Rooij E. The art of microRNA research. Circ Res. 2011:108:219-34.

26. Mendell JT, Olson EN. MicroRNAs in stress signaling and human disease. Cell. 2012;148:1172-87.

27. Park NJ, Zhou H, Elashoff D, Henson BS, Kastratovic DA, Abemayor E, et al. Salivary microRNA: discovery, characterization, and clinical utility for oral cancer detection. Clin Cancer Res. 2009;15:5473-7.

28. Kosaka N, Iguchi H, Ochiya T. Circulating microRNA in body fluid: a new potential biomarker for cancer diagnosis and prognosis. Cancer Sci. 2010;101:2087-92

29. Valadi H, Ekstrom K, Bossios A, Sjostrand M, Lee JJ, Lotvall JO. Exosomemediated transfer of mRNAs and microRNAs is a novel mechanism of genetic exchange between cells. Nat Cell Biol. 2007;9:654-9.

30. Turchinovich A, Samatov TR, Tonevitsky AG, Burwinkel B. Circulating miRNAs: cell-cell communication function? Front Genet. 2013:4:119.

31. McGregor RA, Choi MS. microRNAs in the regulation of adipogenesis and obesity. Curr Mol Med. 2011;11:304-16.

32. Deiuliis JA. MicroRNAs as regulators of metabolic disease: pathophysiologic significance and emerging role as biomarkers and therapeutics. Int J Obes (Lond). 2016;40:88-101.
33. Vlachos IS, Kostoulas N, Vergoulis T, Georgakilas G, Reczko M, Maragkakis M, et al. DIANA miRPath v.2.0: investigating the combinatorial effect of microRNAs in pathways. Nucleic Acids Res. 2012;40(Web Server issue): W498-504.

34. Kanehisa M, Goto S, Sato Y, Kawashima M, Furumichi M, Tanabe M. Data, information, knowledge and principle: back to metabolism in KEGG. Nucleic Acids Res. 2014;42:D199-205.

35. Chen L, Song J, Cui J, Hou J, Zheng X, Li C, et al. microRNAs regulate adipocyte differentiation. Cell Biol Intern. 2013;37:533-46.

36. Zhang R, Wang D, Xia Z, Chen C, Cheng P, Xie H, et al. The role of microRNAs in adipocyte differentiation. Front Med. 2013;7:223-30.

37. Son YH, Ka S, Kim AY, Kim JB. Regulation of adipocyte differentiation via microRNAs. Endocrinol Metab (Seoul). 2014:29:122-35.

38. Linhart HG, Ishimura-Oka K, DeMayo F, Kibe T, Repka D, Poindexter B, et al. C/EBPalpha is required for differentiation of white, but not brown, adipose tissue. Proc Natl Acad Sci U S A. 2001;98:12532-7.

39. Tang YF, Zhang Y, Li XY, Li C, Tian W, Liu L. Expression of miR-31, miR-125b-5p, and miR-326 in the adipogenic differentiation process of adipose-derived stem cells. OMICS. 2009;13:331-6.

40. Li S, Chen X, Zhang H, Liang X, Xiang Y, Yu C, et al. Differential expression of microRNAs in mouse liver under aberrant energy metabolic status. J Lipid Res. 2009:50:1756-65.

41. Novak J, Kruzliak P, Bienertova-Vasku J, Slaby O, Novak M. MicroRNA-206: a promising theranostic marker. Theranostics. 2014:4:119-33.

42. Walden TB, Timmons JA, Keller P, Nedergaard J, Cannon B. Distinct expression of muscle-specific microRNAs (myomirs) in brown adipocytes. J Cell Physiol. 2009;218:444-9.

43. Zhong D, Huang G, Zhang $Y$, Zeng $Y, X u$ Z, Zhao $Y$, et al. MicroRNA-1 and microRNA-206 suppress LXRa-induced lipogenesis in hepatocytes. Cell Signal. 2013;25:1429-37.

44. Bi P, Shan T, Liu W, Yue F, Yang X, Liang XR, et al. Inhibition of Notch signaling promotes browning of white adipose tissue and ameliorates obesity. Nat Med. 2014:20:911-8.

45. Prats-Puig A, Ortega FJ, Mercader JM, Moreno-Navarrete JM, Moreno M, Bonet $\mathrm{N}$, et al. Changes in circulating microRNAs are associated with childhood obesity. J Clin Endocrinol Metab. 2013;98:E1655-60.

46. Can $\mathrm{U}$, Buyukinan $\mathrm{M}$, Yerlikaya $\mathrm{FH}$. The investigation of circulating microRNAs associated with lipid metabolism in childhood obesity. Pediatr Obes. 2015. doi:10.1111/ijpo.12050.

47. Mestdagh P, Hartmann N, Baeriswyl L, Andreasen D, Bernard N, Chen C, et al. Evaluation of quantitative miRNA expression platforms in the microRNA quality control (miRQC) study. Nat Methods. 2014;11:809-15.

48. Cole TJ, Lobstein T. Extended international (IOTF) body mass index cut-offs for thinness, overweight and obesity. Pediatr Obes. 2012;7:284-94.

\section{Submit your next manuscript to BioMed Central and we will help you at every step:}

- We accept pre-submission inquiries

- Our selector tool helps you to find the most relevant journal

- We provide round the clock customer support

- Convenient online submission

- Thorough peer review

- Inclusion in PubMed and all major indexing services

- Maximum visibility for your research

Submit your manuscript at www.biomedcentral.com/submit
) Biomed Central 